\title{
Erratum to: Geometry effect on the estimation of band reflectance in an urban area
}

\author{
C. M. Frey • E. Parlow
}

Published online: 29 September 2011

(C) Springer-Verlag 2011

Erratum to: Theor Appl Climatol (2009) 96:395-406

DOI 10.1007/s00704-008-0048-y

The original version of this article unfortunately contained mistakes.

The definitions used in Eq. 10 in page 7 were corrected as follows:

$M A D=\frac{1}{n} \sum_{i=1}^{n}\left(\left|L_{g_{-} \text {horizontal }}-L_{g i}\right|\right)$

MAD Mean absolute difference

Lgi Global irradiance at $\mathrm{i}^{\text {th }}$ pixel, integrated from wavelength $\lambda_{1}$ to $\lambda_{2}\left[\mathrm{Wm}^{2} \mathrm{sr}^{1} \mu \mathrm{m}^{1}\right]$

Lg_horizontal Global irradiance on a horizontal plane, integrated from wavelength $\lambda_{1}$ to $\lambda_{2}$ $\left[\mathrm{Wm}^{2} \mathrm{sr}^{1} \mu \mathrm{m}^{1}\right.$ ]

Equation 11 in page 8 should appear as follows:

$M D=\frac{1}{n} \sum_{i=1}^{n}\left(L_{g_{-} \text {horizontal }}-L_{g i}\right)$

MD Mean difference

The online version of the original article can be found at http://dx.doi. org/10.1007/s00704-008-0048-y.

C. M. Frey $(\bowtie) \cdot$ E. Parlow

Department of Geosciences, Institute for Meteorology,

Climatology and Remote Sensing, University of Basel,

Klingelbergstrasse 27,

4056 Basel, Switzerland

e-mail: corinne.frey@unibas.ch 